\title{
TREE BASED WAVElET TRANSFORM AND DAG SVM FOR SEIZURE DETECTION
}

\author{
A.S.Muthanantha Murugavel ${ }^{1}$ and S.Ramakrishnan ${ }^{2}$ \\ ${ }^{1}$ Department of Information Technology, Pollachi, Tamilnadu, India \\ as_ananth2k1@yahoo.com \\ ${ }^{2}$ Department of Information Technology, Pollachi, Tamilnadu, India \\ ram_f77@yahoo.com
}

\begin{abstract}
In this paper, we have proposed a new tree based wavelet transform (TBWT) for feature extraction scheme for epileptic seizure detection. Also this paper uses the Directed Acyclic Graph Support Vector Machine (DAGSVM) for the multi-class electroencephalogram (EEG) signals classification. The main aim was to determine the effective features for this problem. Wavelets have played an important role in biomedical signal processing for its ability to capture localized spatial-frequency information of EEG signals. The TBWT works well for high dimensional, multi-class data streams. Decision making was performed in two stages: feature extraction by computing the approximate and detailed wavelet coefficients and classification using the classifiers trained on the extracted features. We have compared the TBWT with wavelet based transform by evaluating with the benchmark EEG dataset. Our experimental results show that the TBWT with DAGSVM gives higher classification accuracy such as $97 \%$ than the existing classifier.
\end{abstract}

\section{KEYWORDS}

Electroencephalogram (EEG) signals classification, epileptic seizure detection, Tree based Wavelet Transform, Directed Acyclic Graph Multi-Class Support Vector Machine (DAGSVM)

\section{INTRODUCTION}

The electroencephalogram (EEG) is a complex and aperiodic time series, which is a sum over a very large number of neuronal membrane potentials. Despite rapid advances of neuroimaging techniques, EEG recordings continue to play an important role in both the diagnosis of neurological diseases and understanding the psychophysiological processes. In order to extract relevant information from recordings of brain electrical activity, a variety of computerizedanalysis methods have been developed. Most methods are based on the assumption that the EEG is generated by a highly complex linear system, resulting in characteristic signal features like nonstationary or unpredictability [1]. Much research with nonlinear methods revealed that the EEG is generated by a chaotic neural process of low dimension [2]-[4]. As in traditional pattern recognition systems, classification of biomedical signals consists of two main modules namely Feature Extraction and Feature Classification. In recent papers, for studying and analyzing the behaviour of EEG signals, chaos theory was used [1]-[4]. To quantify the complexity of EEG signals, numbers of entropy estimators are available. This proposed technique uses wavelet coefficients, which are generated using new generalized tree based wavelet approach as the input feature. The general structure of developed EEG signals classification model has two modules

DOI : $10.5121 /$ sipij.2012.3111 
Signal \& Image Processing : An International Journal (SIPIJ) Vol.3, No.1, February 2012

(Figure.1). A significant contribution of our work is the composition of composite features, which are used to train novel classifier. We use the Directed Acyclic Graph SVM for the classification mechanism. For the classification and comparison we are using the benchmarked datasets (EEG Signals) which includes three classes. The paper is organized as follows. In Section 2, we briefly presented the literature survey that has been performed. In Section 3 we described about the benchmark dataset, our proposed methodology with feature extraction method and classification techniques that are considered. In Section 4, we compared the results of the proposed classifiers using the features with other existing classifier. And Section 5 concludes the paper.

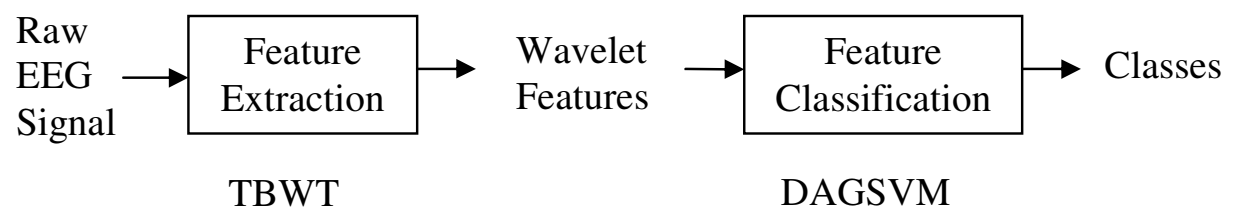

Figure 1. General Structure of developed EEG-signals classification

\section{LiTERATURE SURVEY}

Automatic analysis and diagnosis of epilepsy based on EEG recordings is started in the early 1970s. Today, computer-based analysis addresses two major problems: 1) interictal event detection 2) epileptic seizure analysis [1]. Various feature extraction techniques have been used for the classification of EEG signals with the emphasis on seizure detection. Non-linear based feature extraction technique uses Correlation Dimension, Lyapunov Exponent and Standard Deviation for extracting the features of EEG signals [1][2]. Entropy is a term of thermodynamics that is used to describe amount of disorder in a system. Entropy based technique uses Approximate Entropy (ApEn) as the input feature [4] [6]. Wavelet based technique uses Max, Min, Mean and Standard Deviation [2] [7] [8] [9]. Time Frequency based technique, Feature extraction based on Local Variance [3]. The benchmarked dataset that have been used in the existing works was used to compare the proposed method with the existing methods. The EEG signal classification techniques are divided into three broader categories: Conventional classifiers such as Linear Discriminant Analysis [10], Support Vector Machine [8], and Naïve Bayes [1]. Neural Networks such as MLPNN [8], PNN [10], and RBFNN [6], Combinational classifiers such as Boosting, Voting and Stacking [11].

\section{Proposed Methodology}

\subsection{Dataset Description}

This paper uses the already available benchmark EEG dataset described in Andrzejak et al. [8]. There are three classes (denoted A-C) of datasets each containing 100 single-channel EEG segments. Classes A consisted of signals taken from surface EEG recordings that were carried out on five healthy volunteers. The three classes are described briefly in Table 1 . The healthy volunteers were relaxed in an awake-state with eyes open. These signals are then categorised into class A. Classes B, and C originated from EEG archive of pre-surgical diagnosis. EEGs from five patients were selected, all of whom had achieved complete seizure control after resection of one of the hippocampal formations, which was therefore correctly diagnosed to be the epileptogenic zone. EEGs from five patients were selected, all of whom had achieved complete seizure control after resection of one of the hippocampal formations, which was therefore correctly diagnosed to be the epileptogenic zone. Segments in class B were recorded from within the epileptogenic zone. And set $\mathrm{C}$ only contained seizure activity. 
Signal \& Image Processing : An International Journal (SIPIJ) Vol.3, No.1, February 2012

Table 1: Three classes of EEG dataset

\begin{tabular}{|l|l|}
\hline Class & Description \\
\hline $\mathrm{A}$ & $\begin{array}{l}\text { EEG recordings of five healthy volunteers, } \\
\text { with eyes open }\end{array}$ \\
\hline $\mathrm{B}$ & $\begin{array}{l}\text { Seizure-free intervals, from five patients in the } \\
\text { epileptogenic zone }\end{array}$ \\
\hline $\mathrm{C}$ & $\begin{array}{l}\text { During Seizure intervals, from five patients in } \\
\text { the epileptogenic zone }\end{array}$ \\
\hline
\end{tabular}

\subsection{Extraction of features}

Transforming the input data into the set of features is called feature extraction. Wavelet Transform (WT) is a spectral estimation technique in which any general function can be expressed as an infinite series of wavelets. A major advantage of wavelet methods is their ability to simultaneously localize signal content in both space and frequency. Abnormalities in the EEG in serious psychiatric disorders are many times too subtle to be detected using conventional techniques, such as Fourier transform. We aim at extending the wavelet transform to a generalized tree-based wavelet transform (TBWT). This method is an extension of the Haar like transform introduced by Gavish et al [15], which can also construct data adaptive orthonormal wavelets beyond Haar. This method explains how to construct the data driven-hierarchical tree employed by the extended scheme. The construction of each coarse level of the tree involves finding a path which passes through the set of data points in the finer level. The points order in this path defines the permutation applied to the approximation coefficients of the finer level in the wavelet decomposition scheme.

\subsubsection{Tree-Based Haar Wavelets}

Let $\mathrm{X}=\left\{\mathrm{x}_{1}, \ldots, \mathrm{X}_{\mathrm{N}}\right\}$ be the dataset that contains the different classes of EEG signals, where the samples xi $\epsilon \mathrm{R}^{\mathrm{n}}$ may be points in high dimension, or feature points associated with the nodes of a weighted graph or network. Also, let $f: X \rightarrow R$ be a scalar function defined on the dataset, and let $V=\{f \mid f: X \rightarrow R\}$ be the space of all functions on the dataset. Here we use the following inner product with the space $\mathrm{V}$ :

$$
\langle f, g\rangle=\sum_{j=1}^{N} f\left(x_{j}\right) g\left(x_{j}\right)
$$

which is different from the one used by Gavish et al. [15], since it does not contain a normalizing factor before the sum. We note that when the dataset is a weighted graph, but no feature points are available, the diffusion map framework [16],[17] can be employed in order to obtain such points from the graph. The Euclidean distance between the points produced by this framework approximates the "diffusion distance" [16], [17], which describes the relationship between the graph nodes in terms of their connectivity.

Gavish et al. assume that the geometry and structure of the data $\mathrm{X}$ are captured by one or several hierarchical trees. They do not insist on any specific construction method for these trees, but only that they will be balanced [15]. Given such a tree, a multiscale wavelet-like orthonormal basis for the space $\mathrm{V}$ is to be construced. They start by showing that such a tree induces a multi-resolution analysis with an associated Haar-like wavelet. 


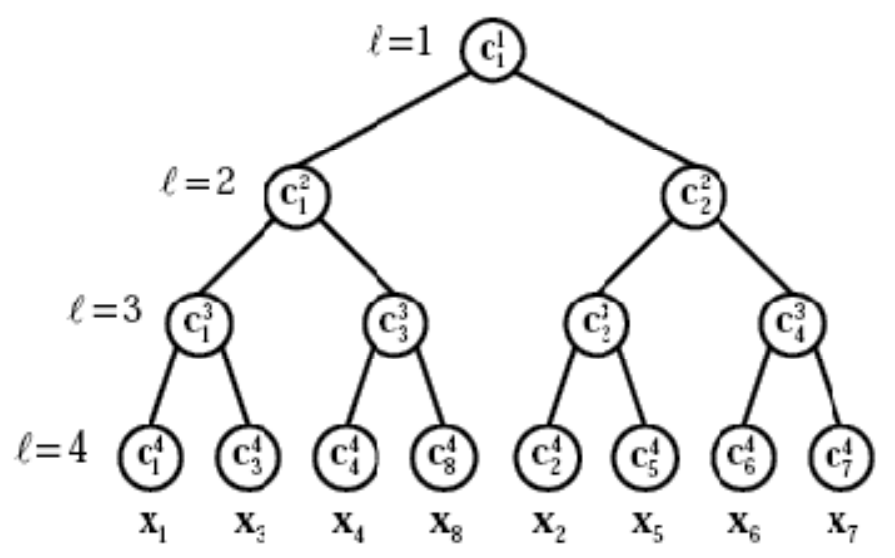

Figure 2. Illustration of a complete full binary tree.

Let $\ell=1, \ldots, \mathrm{L}$ denote the level in the tree, with $\ell=1$ being the root and $\ell=\mathrm{L}$ being the lowest level, where each sample $\mathrm{x}_{\mathrm{j}}$ is a single leaf. Also, let $\mathrm{V}^{\ell}$ denote the space of functions constant on all folders (subtrees) at level $\ell$, and let $1_{\mathrm{X}}$ denote a constant function on $\mathrm{X}$ with the value 1 . Then $\mathrm{V}^{1}=\operatorname{Span}_{\mathrm{R}}\left\{1_{\mathrm{x}}\right\}, \mathrm{V}^{\mathrm{L}}=\mathrm{V}$ and by construction

$V^{1} \subset \ldots \subset V^{\ell} \subset V^{\ell+1} \subset \ldots \subset V^{L}$

Now, let $\mathrm{W}^{\ell}(1 \leq \ell<\mathrm{L})$ be the orthogonal complement of $\mathrm{V}^{\ell}$ in $V^{\ell+1}$. Then, the space of all functions $\mathrm{V}$ can be decomposed as

$$
V=V^{L}=\left[\bigoplus_{\ell=1}^{L-1} W^{\ell}\right] \bigoplus V^{1}
$$

Here we focus on the case of constructing the complete full binary trees and the corresponding orthonormal bases. Let $c_{j}^{\ell}$ denote the j-th point at level $\ell$ of the tree, where $c_{j}^{\ell}=\mathrm{x}_{\mathrm{j}}$, and let $\mathrm{P}_{\ell}$ and $\mathrm{p}_{\ell}$ denote a set and a vector containing point indices, respectively. Also, let $\omega(. ;$. .) be a distance measure in $\mathrm{R}^{\mathrm{n}}$, and let $\mathrm{W}^{\ell}$ be a distance vector associated with the $\ell$-th level of the tree, where $w_{i, j}^{\ell}=w\left(c_{i}^{\ell}, c_{j}^{\ell}\right)$. The distance function describes the first order interaction between data-points, and therefore it should be chosen so as to capture some notion of similarity between them, which would be meaningful to the application at hand. A complete full binary tree can be constructed from the data according to its algorithm which is defined in section 3.2.2. An example for such a tree is shown in Figure. 2.

In the case of a complete full binary tree, the Haar-like basis constructed from the tree is essentially the standard Haar basis which we denote $\left\{\varphi_{j}\right\}_{j=1}^{N}$. The adaptivity of the transform is obtained by the fact that this basis is used to represent a permuted version of the signal $f$, which is more efficiently represented by the Haar basis than $\mathrm{f}$ itself. The permutation is derived from the tree, and is dependent on the data $\mathrm{X}$. Let $\mathrm{p}$ denote a vector of length $\mathrm{N}$, which contains the indices of the points $\mathrm{xj}$, in the order determined by the lowest level of the fully constructed tree. For example, for the tree in Fig. $1, \mathrm{p}=[1 ; 3 ; 4 ; 8 ; 2 ; 5 ; 6 ; 7]^{\mathrm{T}}$. Also let $\mathrm{f}^{\mathrm{p}}$ be the signal $\mathrm{f}$ permuted according to the vector $\mathrm{p}$. The wavelet coefficients can be calculated by the inner products $\left\langle f^{p}, \varphi_{j}\right\rangle$. We hereafter term the scheme described above as tree-based Haar wavelet transform, and we show next that it can be extended to operate with general wavelet filters. 
Signal \& Image Processing : An International Journal (SIPIJ) Vol.3, No.1, February 2012

\subsubsection{Generalized Tree Construction and Wavelet Transform}

In this case the process of building the tree is little different from its previous section. In every level $\ell$ of the tree, we first construct a distance vector $\mathrm{W}^{\ell}$ using the mean points $c_{j}^{\ell}$ and the distance $\omega$.The algorithm for construction of full binary tree is as follows

Given the points $\left\{x_{j}\right\}_{j=1}^{N}$ and the distance function $\omega$.

- Set $c_{j}^{L}=x_{j}$ as the tree leaves

- Perform the following steps for $\ell=L, \ldots, 2$

1. Construct a distance vector $\mathrm{W}^{\ell}$, where $\omega_{i}^{\ell}=\omega\left(c_{i}^{\ell}\right)$

2. Set $\mathrm{P}_{\ell}=\varnothing$

3. Group the points in level $\ell$ in pairs by repeating $N / 2^{1+L-\ell}$ times

- Choose a random point $c_{j 0}^{\ell}, j_{0} \notin P_{\ell}$ and update $\mathrm{P}_{\ell}=\mathrm{P}_{\ell} \mathrm{U}\left\{\mathrm{j}_{0}\right\}$

- Pair $c_{j 0}^{\ell}$ with the point $c_{j 1}^{\ell}$

- Update $\mathrm{P}_{\ell}=\mathrm{P}_{\ell} \mathrm{U}\left\{\mathrm{j}_{1}\right\}$.

4. Place in a vector $\mathrm{p}_{\ell}$, the reordered point indices of $\mathrm{P}_{\ell}$

5. Construct the coarse level $\ell-1$ from the finer level $\ell$ by replacing each pair $c_{i}^{\ell}$ and $c_{j}^{\ell}$ with the mean point $1 / \sqrt{2}\left[c_{i}^{\ell}+c_{j}^{\ell}\right]$

The above algorithm yields the tree node points $c_{j}^{\ell}$ and the vectors $\mathrm{p}_{\ell}$ containing the points order in each tree level as its output.

Next let $\mathrm{f}=\left[\mathrm{f}\left(\mathrm{x}_{1}\right), \ldots, \mathrm{f}\left(\mathrm{x}_{\mathrm{N}}\right)\right]^{\mathrm{T}}$, and let $\mathrm{a}_{\ell}$ and $\mathrm{d}_{\ell}$ denote the approximation and detail coefficient vectors, respectively, received for $\mathrm{f}$ at level $\ell$, where $\mathrm{a}_{\mathrm{L}}=\mathrm{f}$. Also, let $\mathrm{P}_{\ell}$ denote a linear operator that reorders a vector according to the indices in $\mathrm{p}_{\ell}$. Then, applying the Haar transform derived from the tree to $f$ can be carried out according to the decomposition algorithm which is as follows Given the signal $\mathrm{f}$, the vectors $\left\{p_{\ell}\right\}_{\ell=2}^{L}$, and the filters h' and g'

1. Set $a_{L}=\mathrm{f}$

2. Perform the following steps for $\ell=\mathrm{L}, \ldots ., 2$

- Construct the operator $\mathrm{P}_{\ell}$, that reorders its input vector according to the indices in $\mathrm{p}_{\ell}$

- Apply $\mathrm{P}_{\ell}$ to $\mathrm{a}_{\ell}$ and receive $a_{\ell}^{p}$

- Filter $a_{\ell}^{p}$ with h' and decimate the result by 2 to receive $a_{\ell-1}$.

- Filter $a_{\ell}^{p}$ with g' and decimate the result by 2 to receive $d_{\ell-1}$.

This algorithm yields the approximate coefficient $\mathrm{a}_{1}$ and detail coefficients $\left\{d_{\ell}\right\}_{\ell=1}^{L-1}$ as its output corresponding to signal $\mathrm{f}$.

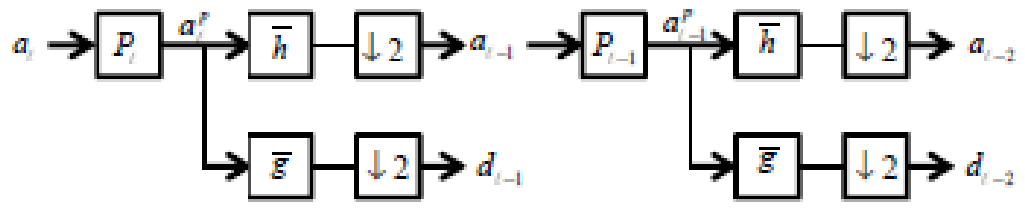

Figure 3. Two single level tree based decomposition steps

Figure. 3 describes two single-level decomposition steps carried out according to decomposition algorithm. We note that here the adaptivity of the transform is related to the permutations applied 
Signal \& Image Processing : An International Journal (SIPIJ) Vol.3, No.1, February 2012

to the coefficients $a_{\ell}$ in every level of the tree. In fact, without the operator $\mathrm{P}_{\ell}$ applied in each level, the decomposition scheme of decomposition algorithm reduces to that of the common 1D orthogonal wavelet transform. Also, since permutation of points is a unitary transform, the described transform remains unitary. The EEG signals can be considered as a superposition of different structures occurring on different time scales at different times. Selection of appropriate wavelet and the number of decomposition levels is very important in the analysis of signals using the WT.

\subsection{Classifiers used for Classification}

\section{Directed Acyclic Graph Support Vector Machine(DAGSVM)}

Support Vector Machines (SVMs) [1] are very popular and powerful in learning systems because of attending high dimensional data, providing good generalization properties, their ability to classify input patterns with minimized structural misclassification risk. In two ways we can have a multi-class SVM classifier; one is to directly consider all data in one optimization formulation, and the other is to decompose multi-class problem to several binary problems. The second solution is a better idea and has been considered more than the first approach [13-17] because binary classifiers are easier to implement and moreover some powerful algorithms such as Support Vector Machine (SVM) are inherently binary [18]. Two major decomposition implementations are: "one-against-all" and "one-against one".

The one-against-all [1] method constructs $\mathrm{n}$ SVMs where $\mathrm{n}$ is the number of classes. The i-th SVM is trained to separate the i-th class from remaining classes. The one-against-one [14] (pairwise SVMs) instead, constructs $n(n-1) / 2$ decision functions for all the combinations of class pairs. In determination of a decision function for a class pair, we use the training data for the corresponding two classes. Thus, in each training session, the number of the training data is reduced considerably compared to one-against-all support vector machines, which use all the training data. Experimental results in [19] indicate that the one-against-one is more suitable for practical use.

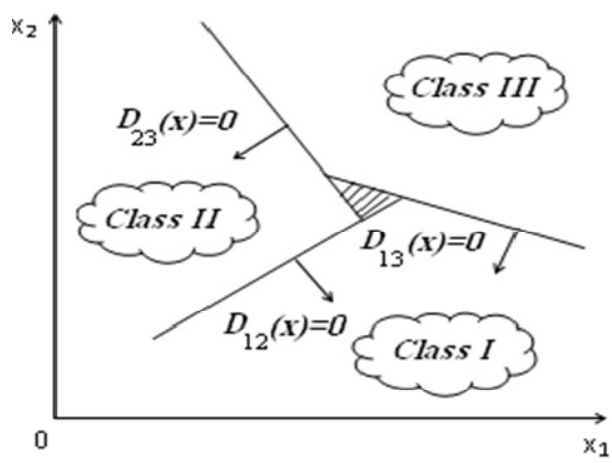

Figure 4. Unclassifiable regions formed by pairwise function.

A problem with both on-against-all and pair wise support vector machines is unclassifiable regions . Figure 4 shows the unclassifiable problem for three classes. To resolve this problem, Vapnik proposed to use continuous decision functions. Namely, we classify a datum into the class with maximum value of the decision functions. Inoue and Abe proposed fuzzy support vector machines, in which membership functions are defined using the decision functions. Another popular solution is DAG SVM that uses a decision tree in the testing stage. Training of a DAG is the same as conventional pair wise SVMs. Classification by DAGs is faster than by conventional 
pairwise SVMs or pairwise fuzzy SVMs. The decision tree for three class DAGSVM is shown in Figure 5.

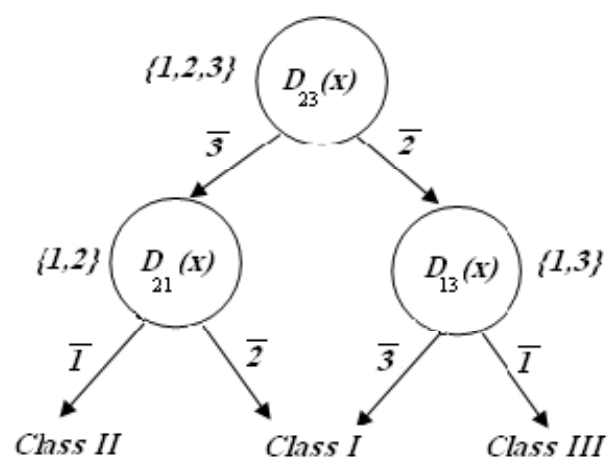

Figure 5. DAG Classification

The generalization regions become as shown in Figure 6. Unclassifiable regions are resolved, but clearly the generalization regions depend on the tree formation.

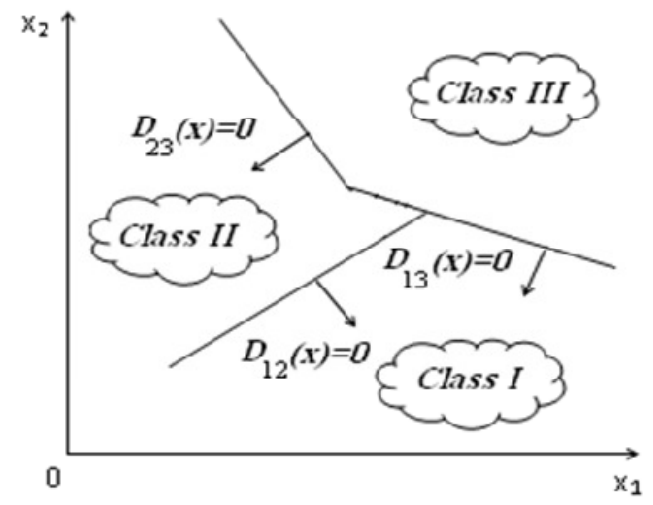

Figure 6. Generalization region by DAG classification

\section{Results and Discussion}

In our work, we employed a tree based wavelet transform for extracting wavelet coefficients from the dataset in order to extract transient features. The wavelet base features possess good characteristics such as robustness in the characterization of the epileptic patterns and low computational burden. Wavelet based features were computed for both normal and epileptic EEG signals and are fed as inputs to the three classifiers such as PNN, RBFNN and DAGSVM. Figure 7 compares between-class-distance and within-class-distance for the three classes of datasets based on the tree based wavelet features. From the figure, it was observed that the within-classdistance was minimum and the between-class-distance was maximum. So the extracted features are well suited for discriminating various classes. Figure 8 shows EEG Signal Patterns based on the wavelet features. For the classification of EEG signals we have used a DAG SVM. Among the available 100 EEG data sets, 50 data sets are used for training and the remaining data sets are used for testing the performance of the neural network and SVM classifiers. Computational cost and the classification time for the proposed DAGSVM classifier depend on the number of support vectors required for the design of the classifier and the kernel employed. Increase in number of support vectors lead to increase in computational requirements. It is shown that our wavelet based 
Signal \& Image Processing : An International Journal (SIPIJ) Vol.3, No.1, February 2012

features possesses good characteristics such as robustness in the characterization of the epileptic patterns and low computational burden. The Classification and misclassification results of various EEG datasets represented by the features are given in Table 2. The performance in the rate of classification of the two classifiers such as PNN, RBFNN and DAG SVM have been and which is shown in Table 3. The Multi-class DAGSVM using wavelet based features gives superior performance that is $97 \%$ in terms of classification rate.

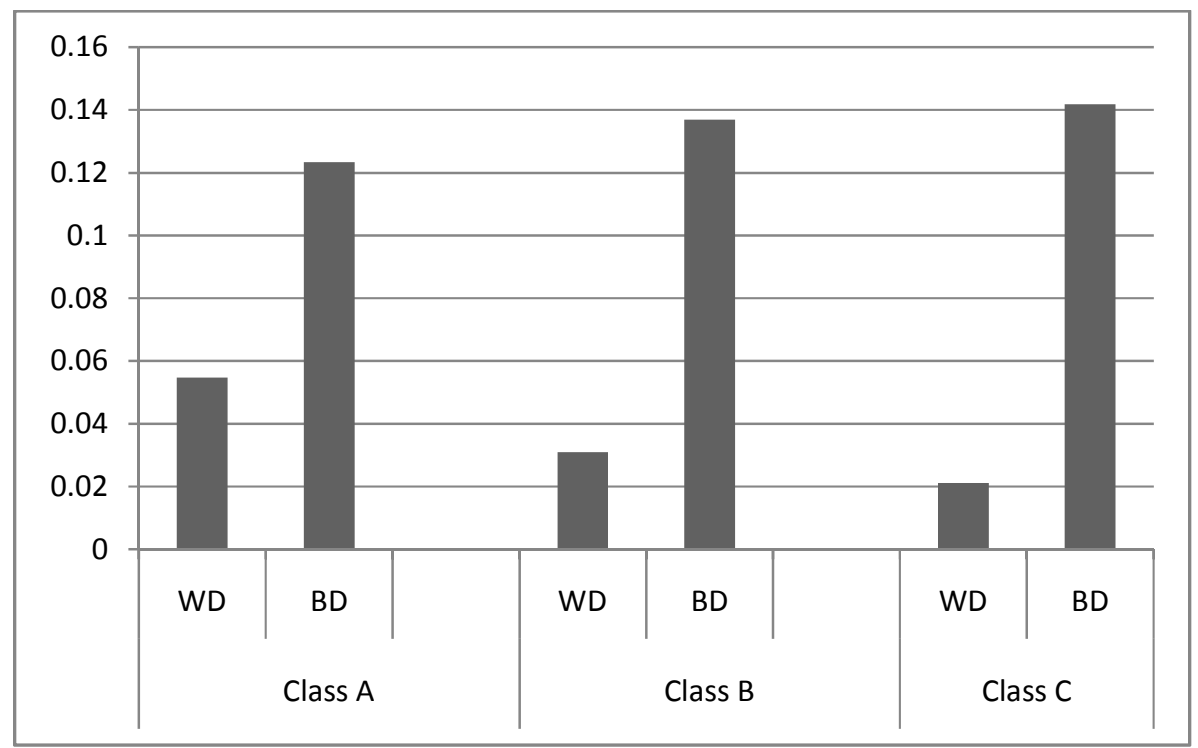

Figure 7. Comparison of between-class-distance and within-class-distance for various hierarchical classes based on the features

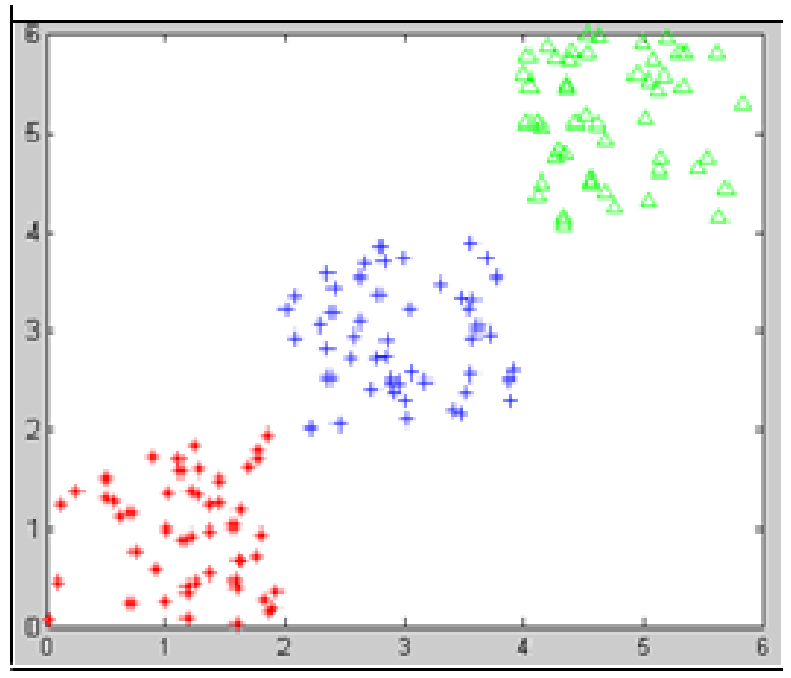

Figure 8. EEG Signal Patterns based on the wavelet features 
Signal \& Image Processing : An International Journal (SIPIJ) Vol.3, No.1, February 2012

Table 2. Classification and Misclassification Results of Various SVMs

\begin{tabular}{cccc}
\hline Dataset & Size & \multicolumn{2}{c}{ Output Results } \\
\cline { 3 - 4 } & & $\begin{array}{c}\text { Correctly } \\
\text { classified }\end{array}$ & $\begin{array}{c}\text { Mis- } \\
\text { classified }\end{array}$ \\
\hline$\{$ A,B,C $\}$ & 150 & 146 & 5 \\
\hline A,B $\}$ & 100 & 97 & 3 \\
\hline$\{\mathrm{A}, \mathrm{C}\}$ & 100 & 50 & 2 \\
\hline
\end{tabular}

Table 3. Comparison of classifiers such as PNN, RBFNN and DAGSVM

\begin{tabular}{|l|c|c|c|}
\hline Classifier & PNN & RBFNN & DAGSVM \\
\hline $\begin{array}{l}\text { Overall } \\
\text { Classification } \\
\text { Accuracy }\end{array}$ & $94 \%$ & $93 \%$ & $97 \%$ \\
\hline
\end{tabular}

\section{Conclusion}

The Multi-class DAG SVM has shown great performance since it measures the predictability of the current amplitude values of a physiological signal based on its previous amplitude values. A robust and computationally low-intensive wavelet based features have been used for the proposed EEG classification system. Besides this, the PNN provided encouraging results, which could have originated from the architecture of the PNN. The performance of the other neural network was not as high as the DAGSVM. The results of the present paper demonstrated that the DAGSVM with the tree based wavelet features can well preserve the most discriminant information of EEG signals and improve the performance. In current work, focus was put on normal, seizure-free intervals and during seizure intervals. In the next stage of research, the results from this preliminary study will be expanded to include a more complete range of datasets.

\section{ACKNOWLEDGMENT}

The authors wish to thank Andrzejak et al., 2001 for the benchmark EEG dataset available: (http://www.meb.unibonn.de/epileptologie/science/physik/eegdata.html).

\section{REFERENCES}

[1] Hojjat Adeli, Member, IEEE, Samanwoy Ghosh-Dastidar, and Nahid Dadmehr. :A Wavelet-Chaos Methodology for Analysis of EEGs and EEG Subbands to Detect Seizure and Epilepsy. IEEE Transactions on Biomedical Engineering, Vol. 54, No. 2 (2007)

[2] Samanwoy Ghosh-Dastidar, Hojjat Adeli, Member, IEEE, and Nahid Dadmehr.: Mixed-Band Wavelet-Chaos- Neural Network Methodology for Epilepsy and Epileptic Seizure Detection. IEEE Transactions on Biomedical Engineering, Vol. 54, No. 9 (2007)

[3] Samanwoy Ghosh-Dastidar, Hojjat Adeli, Member, IEEE, and Nahid Dadmehr.: Principal Component Analysis-Enhanced Cosine Radial Basis Function Neural Network for Robust Epilepsy and Seizure Detection. IEEE Transactions on Biomedical Engineering, Vol. 55, No. 2 (2008) 
Signal \& Image Processing : An International Journal (SIPIJ) Vol.3, No.1, February 2012

[4] Vairavan Srinivasan, Member, IEEE, Chikkannan Eswaran, Senior Member, IEEE, and Natarajan Sriraam, Member, IEEE.: Approximate Entropy-Based Epileptic EEG Detection Using Artificial Neural Networks. IEEE Transactions on Information Technology in Biomedicine, Vol. 11, No. 3 (2007)

[5] Alexandros T. Tzallas, Member, IEEE, Markos G. Tsipouras, and Dimitrios I. Fotiadis, Senior Member, IEEE.: Epileptic Seizure Detection in EEGs Using Time-Frequency Analysis. IEEE Transactions on Information Technology in Biomedicine, Vol. 13, No. 5 (2009)

[6] Pari Jahankhani, Vassilis Kodogiannis and Kenneth Revett.: EEG Signal Classification Using Wavelet Feature Extraction and Neural Networks," IEEE John Vincent Atanasoff 2006 International Symposium on Modern Computing (JVA'06) 0-7695-2643-8/06 (2006)

[7] A.S. Muthanantha Murugavel, and S. Ramakrishnan.: Classification of EEG Signals Using Weighted Probabilistic Neural Network and Multiwavelet Transformation. Proceedings of Proceedings of the International Conference on Operation Research applications in Engineering and Management (ICOREM), May 27-29 (2009)

[8] Hiram Firpi, Member, IEEE, Erik D. Goodman, and Javier Echauz, Member, IEEE.: Epileptic Seizure Detection Using Genetically Programmed Artificial Features. IEEE Transactions on Biomedical Engineering, Vol. 54, No. 2 (2007)

[9] Inan Gu"ler and Elif Derya U* beyli.: Multiclass Support Vector Machines for EEG-Signals Classification. IEEE Transactions on Information Technology In Biomedicine, Vol. 11, No. 2 (2007)

[10] Deon Garrett, David A. Peterson, Charles W. Anderson.: Comparison of Linear, Nonlinear, and Feature Selection Methods for EEG Signal Classification. IEEE Transactions on Neural Systems and Rehabilitation Engineering, Vol. 11, No. 2 (2003)

[11] Neep Hazarika, Ah Chung Tsoi, Senior Member, IEEE, and Alex A. Sergejew.: Nonlinear Considerations in EEG Signal Classification. IEEE Transactions on Signal Processing, Vol. 45, No. 4 (1997)

[12] Ernst Haselsteiner and Gert Pfurtscheller.: Using Time-Dependent Neural Networks for EEG Classification. IEEE Transactions on Rehabilitation Engineering, Vol. 8, No. 4 (2000)

[13] George Townsend, Bernhard Graimann, and Gert Pfurtscheller, Member, IEEE.: Continuous EEG Classification During Motor Imagery-Simulation of an Asynchronous BCI. IEEE Transactions on Neural Systems and Rehabilitation Engineering. Vol. 12, No. 2 (2004)

[14] Piotr Suffczynski, Fernando H. Lopes da Silva, Jaime Parra, Demetrios N. Velis, Brigitte M. (Gitte) Bouwman, Clementina M. van Rijn, Peter van Hese, Paul Boon, Houman Khosravani, Miron Derchansky, Peter Carlen, and Stiliyan Kalitzin.: Dynamics of Epileptic Phenomena Determined From Statistics of Ictal Transitions. IEEE Transactions on Biomedical Engineering, Vol. 53, No. 3 (2006)

[15] M. Gavish, B. Nadler, and R. R. Coifman, "Multiscale wavelets on trees, graphs and high dimensional data: Theory and applications to semi supervised learning," in Proceedings of the 27th International Conference on machine Learning, 2010.

[16] S. Lafon, Y. Keller, and R. R. Coifman, "Data fusion and multicue data matching by diffusion maps," IEEE Transactions on pattern analysis and machine intelligence, vol. 28, no. 11, pp. 17841797, 2006.

[17] R. R. Coifman and S. Lafon, "Diffusion maps," Applied and Computational Harmonic Analysis, vol. 21, no. 1, pp. 5-30, 2006. 


\section{Authors}

A.S. Muthanantha Murugavel is working as an Assistant Professor in department of Information Technology, Dr.Mahalingam College of Engineering and Technology, Pollachi since 2001. Previously he was working as a Software Engineer for 3 years. He completed his Bachelor of Engineering in Computer Science and Engineering from Madurai Kamaraj University in the year 1996 and he completed his Masters of Engineering in Computer Science and Engineering from Anna University, Chennai in the year 2007. Currently he is pursuing Ph.D. in Anna University of Technology, Coimbatore. He has published papers in seven international conferences and six

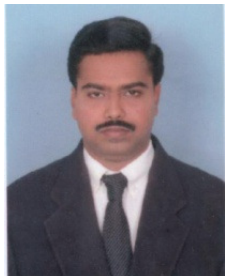
national conferences. His area of interest includes Bio Medical Signal Processing, Web and Multimedia Technologies.

Srinivasan Ramakrishnan received the B.E. degree in Electronics and Communication Engineering from the Bharathidasan University, Trichy, and the M.E. degree in Communication Systems in from the Madurai Kamaraj University, Madurai. $\mathrm{He}$ received his $\mathrm{PhD}$ degree in Information and Communication Engineering from Anna University, Chennai. He has 11 years of teaching experience and 1 year industry experience. $\mathrm{He}$ is a Professor and the Head of the Department of Information Technology, Dr.Mahalingam College of Engineering and Technology, Pollachi, India. Dr.Ramakrishnan is a Reviewer of 14 International Journals such as IEEE

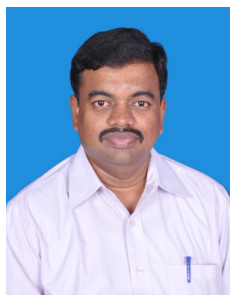
Transactions on Image Processing, IET Journals(Formally IEE), ACM Reviewer for Computing Reviews, Elsevier Science, International Journal of Vibration and Control, IET Generation, Transmission \& Distribution, etc. He is in the editorial board of 6 International Journals. He is a Guest Editor of special issues in 3 International Journals including Telecommunication Systems Journal of Springer. He has published 49 papers in international, national journals and conference proceedings. Dr.S.Ramakrishnan has published a book for LAP, Germany. He has also reviewed 2 books for McGraw Hill International Edition and 1 book for ACM Computing Reviews. He is guiding $8 \mathrm{PhD}$ research scholars. His areas of research include digital image processing, soft computing, human-computer interaction and digital signal processing. 